\title{
Model and Simulation of Globoidal Cam Mechanisms with Oscillating and Dual Stopping Followers
}

\author{
Rongyu Ge , Jin Wang and Chengliang Zhang \\ University of Jinan, School of Mechanical Engineering, 250021 Jinan, China
}

\begin{abstract}
Globoidal cam mechanisms with oscillating and dual stopping followers are necessary to accomplish the rise-dwell-fall-dwell periodic motion. The trajectory surface of roller axis of oscillating globoidal cam mechanism is the offset surface of working surface of the cam, and its mathematical model is relatively simple. According to the spatial geometric relationship, the coordinate equation of trajectory surface of roller axis is derived and the original angle positions for dual stopping followers are analyzed. As a result, the three-dimension (3D) model of globoidal cam mechanism with oscillating and dual stopping followers is generated based on the offset surface method based on the UG software. Finally, the dynamic model is realized using Adams software, and through the dynamic simulation, the oscillating angle displacement, angular velocity and angular acceleration of the double-stop swinging cam mechanism are studied. According to the simulation results, it can be concluded that the input speed of globoidal cam mechanism is an important factor that affects the output performance of the mechanism. Through the above research, it can provide some reference for the design of spatial cam mechanism.
\end{abstract}

\section{Introduction}

The globoidal cam mechanism, including driving cam mounted through input axis and following turret fixed through output shaft, which use physical parts to convert constant rotary motion of input shaft into intermittent motion of output shaft. Due to the advantages of compact structure, high load capacity, high reliability, low noise and low vibration, globoidal cam mechanisms, are widely used in many types of automatic machinery such as machine tool changer, automatic assembly lines, medicine machines, paper processing machines and packing machines [1]. So globoidal cam mechanisms have already become the most popular and precise intermittent device at present. In term of the shape, globoidal cam mechanisms is one of the most complicate, which can be classified cam mechanisms with indexing followers or with oscillating followers according to the output motion. Cam mechanisms with oscillating followers can arrive the reciprocating and oscillating output motion that is used to convey materials between two stations in production line. As is well known, the motion should be stopped for a portion of a cycle while an operation is performed on the production at every station, so globoidal cam mechanisms with oscillating and dual stopping followers are necessary to accomplish the rise-dwell-return-dwell periodic motion.

The most important task when designing and machining the globoidal cam mechanisms is to represent their working surfaces. The working surface of globoidal cams are the surfaces that contact with the roller surfaces.
These surfaces are very complex and it is very difficult to directly create the. Up to now, a number of works dealing with finding the way to describe accurately these surfaces have been proposed. Mostly, researchers derived mathematically expressions for the surface geometry of the globoidal cam based on coordinate transformation, differential geometry, and theory of conjugate surfaces [2-6]. In that way, it is so complex to infer the equations requiring designers to have solid mathematical knowledge. While cam rotates, there is a swept surface of roller axis, which is called the trajectory surface of roller axis in this paper and it is the offset surface of working surface of the globoidal cam. In fact, from designing and machining point of view, the globoidal cam mechanism can be determined by the trajectory surface of roller axis. The objective of this paper is to introduce an effective modelling and analysing method for globoidal cam mechanism with oscillating and dual stopping followers, which can be implemented by using commercial CAD/CAM/CAE softwares.

\section{Mathematical model of trajectory sur- face of roller axis}

\subsection{Calculation of the surface equation}

The structure diagram of globoidal cam mechanism with oscillating and dual stopping followers is shown in Fig. 1. Here, the center distance between the globoidal cam and the oscillating turret is $C$, the pitch radius of oscillating 
turret is $R_{e}$, the roller radius is $R$, the roller length is $m$, the clearance between the bottom end of the roller and the cam body is $e$, the angle between the two roller axes is $\beta$, and $P$ represents any trajectory point on the trajectory surface of roller axis, and the distance from $P$ to the oscillating turret axis is $l$. The coordinate system oxyz is attached on the cam, where the axis of globoidal cam is defined as the $o z$ coordinate axis, the coordinate plane oxy passes through the axis of oscillating turret, and the coordinate axis oy represents the projected line on the coordinate plane oxy of the initial line during the oscillation period. In one moment, the angular displacement of globoidal cam is $\theta_{i}$, when the oscillating angular displacement of lower roller is $\phi^{1}$ and that of upper roller is $\phi^{2}$. As is shown in Fig. 1, the angular displacement of rollers can be obtained as follows:

$$
\begin{gathered}
\phi^{j}=\phi_{0}^{j}+p \cdot \phi_{i} \quad j=1,2 \\
\phi_{i}=\left\{\begin{array}{cc}
S \cdot \phi_{f} & \text { oscillating period } \\
\phi_{f} & \text { stopping period }
\end{array}\right.
\end{gathered}
$$

where, $\phi_{0}^{j}$ is the initial angular displacement of rollers, $\phi_{i}$ denotes the oscillating angular displacement of rollers relative to the cam rotation angle $\theta_{i}, \phi_{f}$ is the oscillating angle range of the oscillating turret, $S$ is the dimensionless angular displacement of oscillating turret, which is decided by various predetermined cam motion curves. $p$ is a symbol coefficient, and when the oscillating turret makes a clockwise rotation, $p$ is equal to 1 , otherwise that is equal to -1 .

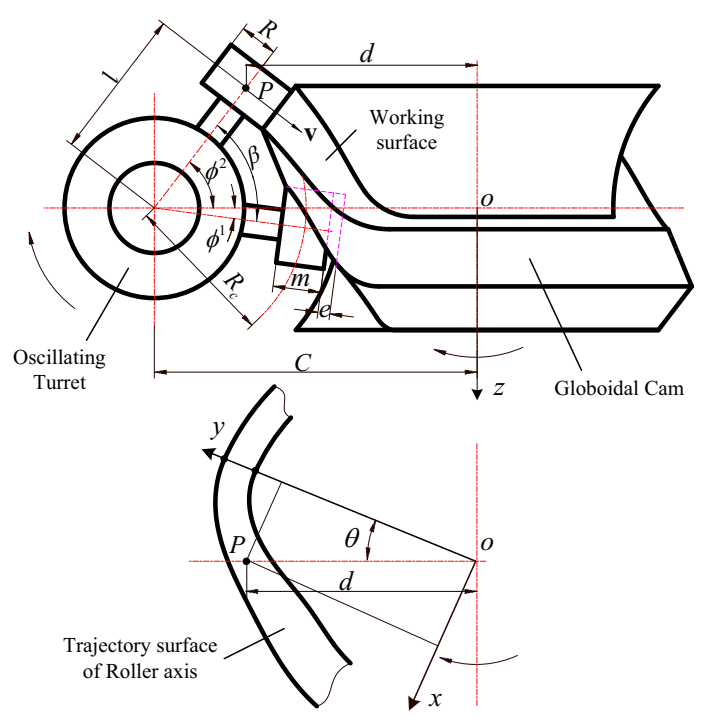

Figure 1. Structure diagram of globoidal cam mechanism

From Fig. 1, the distance $d$ between the trajectory point $P$ of roller axis and the coordinate axis $o z$ can be expressed as:

$$
d=C-l \cos \phi^{j}
$$

Based on the geometry relations as shown in Fig. 1, the coordinate equation of trajectory surface of roller axis in the coordinate system oxyz can be maintained as follows:

$$
\mathbf{r}(l, \theta)=\left[\begin{array}{c}
\left(C-l \cos \phi^{j}\right) \sin \theta \\
\left(C-l \cos \phi^{j}\right) \cos \theta \\
l \sin \phi^{j}
\end{array}\right] \quad j=1,2
$$

Eq. (4) can also be described as

$$
\mathbf{r}(l, \theta)=\left[\begin{array}{c}
C \sin \theta \\
C \cos \theta \\
0
\end{array}\right]+l \cdot\left[\begin{array}{c}
-\cos \phi^{j} \sin \theta \\
-\cos \phi^{j} \cos \theta \\
\sin \phi^{j}
\end{array}\right] \quad j=1,2
$$

From Eq. (5), the equation of trajectory surface of roller axis accords with mathematical definition of the ruled surface:

$$
r(l, \theta)=p(\theta)+l q(\theta)
$$

So, the trajectory surface of roller axis of oscillating globoidal cam is a ruled surface. And the working surface is the offset surface of the trajectory surface of roller axis, but its derivation process of mathematical model is especially complex.

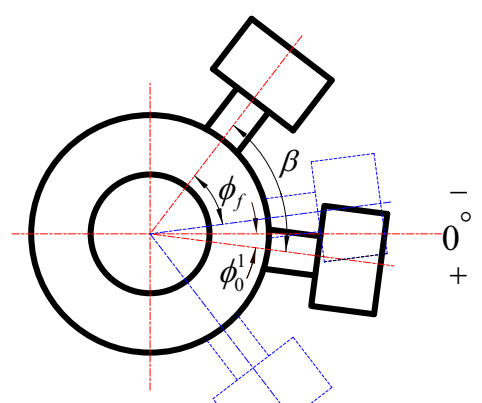

Figure 2. Schematic diagram of initial angle analysis

\subsection{Analysis of initial angular displacement}

For the globoidal cam mechanisms with oscillating and dual stopping followers, the convex body of two intermittent sections is commonly symmetrical structure, and it means that the oscillating bottom dead point of upper roller and the oscillating top dead point of lower roller are symmetric along the coordinate plane of oxy. As is shown in Fig. 2, the central plane oxy denotes the angular displacement with zero degree, and the clockwise is the positive direction. Then when both the two rollers are located in the upper dead point, they are considered with the initial angular displacement. According to the geometry relations, the initial angular displacement of two followers can be given by

$$
\phi_{0}^{j}=\frac{(-1)^{j-1} \beta-t \cdot \phi_{f}}{2} \quad j=1,2
$$


where $t$ represents the symbol coefficient. For the oscillating section and intermittent section shown on the left side of Fig. 2 when two rollers are located in the top dead point, $t$ is equal to 1 . For the other oscillating section and intermittent section when two rollers are located in the bottom dead point, $t$ is equal to -1 .

\section{3D solid modeling based on UG}

Working surface equation of the globoidal cam with oscillating followers is especially complex, so that it is difficult to directly establish its mathematical model in 3D software. At present, many kinds of advanced 3d softwares are integrated with the modeling method based on the offset surface, and in this way precise modeling on the complex regular surface can be indirectly realized. Here, UG software is used as a tool for the model of the globoidal cam mechanism with oscillating followers. The design parameters about the globoidal cam mechanism with oscillating follower is listed as follows: $C=45$, $R=4, R_{c}=22, m=5, e=1, \beta=45^{\circ}, \phi_{f}=20^{\circ}$. The motion cycle of oscillating followers incules four phrase, clockwise oscillation, dwell, anticlockwise oscillation, dwell and the corresponding cam angles are respectively $60^{\circ}$, $60^{\circ}, 135^{\circ}$ and $105^{\circ}$. That is, the rotating angles of globoidal cam during two oscillating period are $\theta_{f 1}=60^{\circ}$ and $\theta_{f 2}=135^{\circ}$. And the widely used modified sine curve is chosen as the cam motion curve.

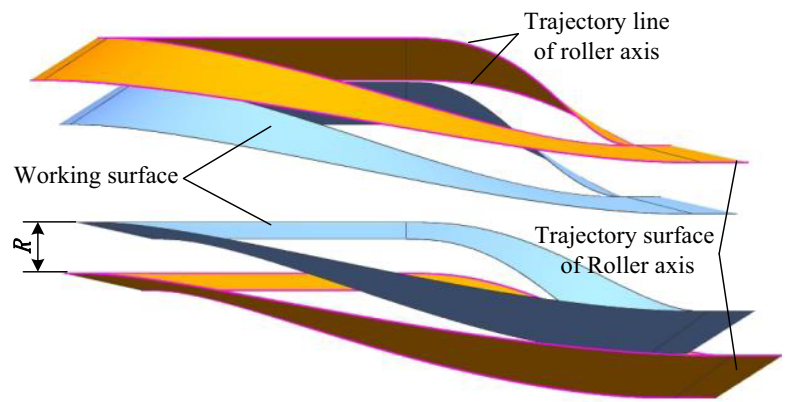

Figure 3. Model of trajectory surface of roller axis and working surface of globoidal cam mechanism

According to Eq. (4), if the parameter $l$ is defined as a fixed value, then $\mathbf{r}(\theta)$ denotes a piece of spatial curve which is called trajectory curve of roller axis in this paper. Actually the trajectory surface of roller axis is an ruled surface that is generated by the sweeping of roller axis along two trajectory curve of roller axis. The working surface of the cam is the offset surface of ruled surface. Based on this idea, parameter $l$ is respectively defined as two fixed value which is expressed as follows:

$$
\begin{aligned}
& l_{1}=R_{e}+m / 2+e \\
& l_{2}=R_{e}-m / 2
\end{aligned}
$$

First of all, a UG expression table should be built including all the constants, variables and equations to accomplish the parametric modeling. Then by using the command 'By law curve' in the curve menu of UG software, two trajectory curves of roller axis are created according to Eq. (4), Eq. (8) and Eq. (9). Besides, using the command 'Offset surface' in the insert menu, two working are generated by offsetting two trajectory surfaces of roller axis with the offset distance roller radius $R$ as is shown in Fig.3. In the same way, the inside surface and outside surface that is placed between the two working surfaces. Subsequently, the two working surfaces, the inside and outside surface are united together to the convex body of globoidal cam by using the command 'Sew'. Cam basis body is obtained by revolution. Eventually, the 3D model of globoidal cam mechanisms with oscillating and dual stopping followers is completed after the boolean operation to unite the basis body and convex body shown in Fig. 4.

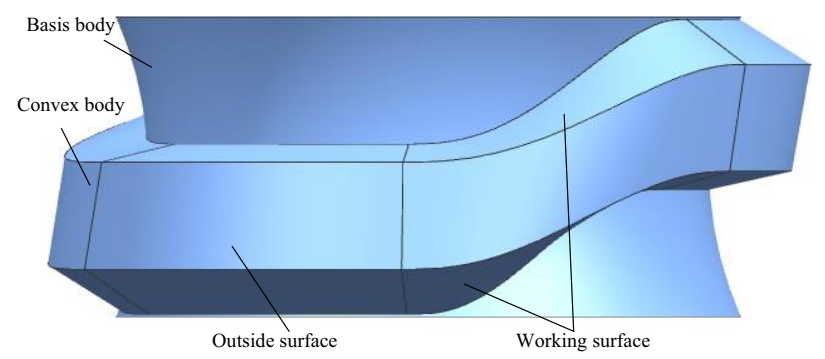

Figure 4. 3D model of oscillating globoidal cam

\section{Dynamic simulation based on ADAMS}

\subsection{Construction of dynamic model}

With MSC Adams software users can produce virtual prototypes to simulate complex mechanical systems and quickly analyze multiple design variations until an optimal design is achieved. MSC Adams is one of many computer programs for modeling and simulating multi body systems. To get the virtual prototype of cam mechanism, the 3D model of the oscillating turret with two rollers is established in the UG software and then assembled with the globoidal cam. The assembling file is saved as *.x_t parasolid file format and imported into ADAMS software.

Using ADAMS, firstly revolute joint (Two bodiesOne Location, Normal To Grid) between the cam and the ground is built, and then revolute joint (Two bodies-One Location, Normal To Grid) between the oscillating turret and output axis is built, adding the rotary pair to the oscillating globoidal cam and the oscillating turret, so that it can rotate freely around the axis of rotation. And then adding the contact force between the cam and the oscillating turret. It is important and complex to create the contact force between the cam and the rollers.

For the software of ADAMS, the contact problem between two rigid bodies is looked as a nonlinear system with a damper and a spring. The contact force $F_{n}$ can be calculated as follows [7]:

$$
F_{n}=K \delta^{e}+C \dot{\delta}
$$


where, $\delta$ and $\dot{\delta}$ are respectively the contact deformation and the deformation velocity, $K$ is contact stiffness, $C$ is damping coefficient, $e$ is rigidity index. For the metal collision, $e$ is equal to 1.5 generally. Contact stiffness $K$ can be expressed by

$$
\begin{aligned}
& K=\frac{4}{3} R^{\frac{1}{2}} E^{*} \\
& \frac{1}{R}=\frac{1}{R_{1}}+\frac{1}{R_{2}}
\end{aligned}
$$

where, $R_{1}$ and $R_{2}$ are the equivalent radius of contact point with the cam and roller. $E^{*}$ can be calculated according to the following equation:

$$
\frac{1}{E^{*}}=\frac{1-\mu_{1}^{2}}{E_{1}}+\frac{1-\mu_{2}^{2}}{E_{2}}
$$

$\mu_{1}$ and $\mu_{2}$ are the poisson ratio with the material of the cam and the roller in this formula. $E_{1}$ and $E_{2}$ are the elastic modulus with the material of the cam and the roller.

ADAMS software uses the step function to define the damping coefficient. The formula is as follows [8]:

$$
C=\operatorname{step}\left[\delta, 0,0, d, C_{\max }\right]
$$

where $d$ is penetrating depth. When the contact stiffness, stiffness coefficient, damping coefficient, infiltration depth are inputted, the dynamic simulation model of globoidal cam mechanism with oscillating is completed, as is shown Fig. 5.

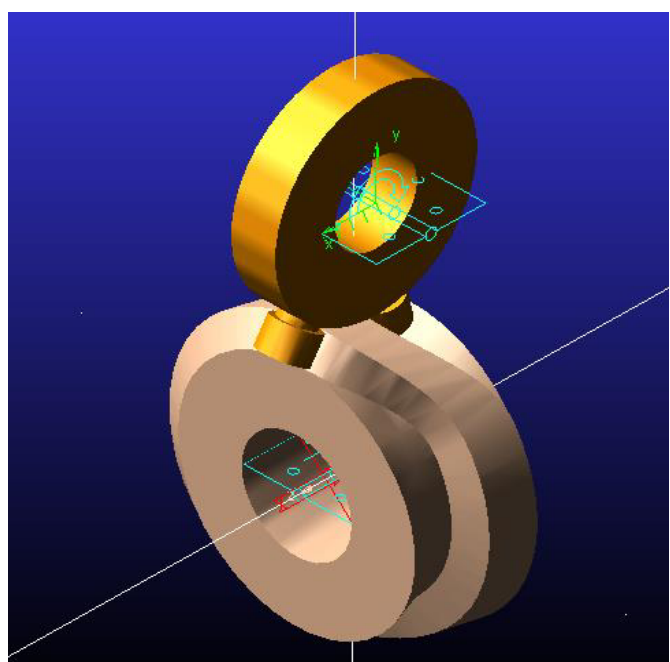

Figure 5. The dynamic simulation model of oscillating globoidal cam mechanism

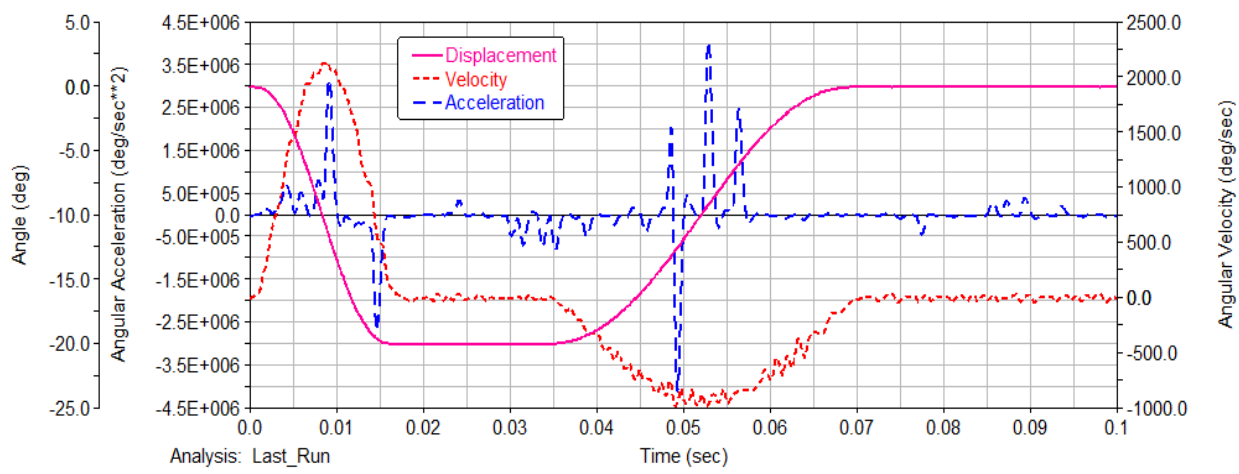

Figure 6. Output curve of angular displacement, angular velocity and angular acceleration of oscillating turret with cam rotation speed $600 \mathrm{r} / \mathrm{min}$

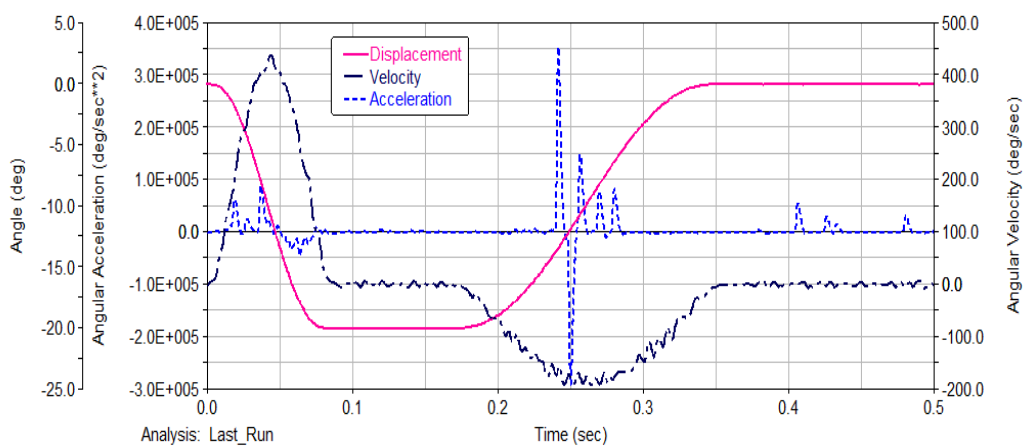

Figure 7. Output curve of angular displacement, angular velocity and angular acceleration of oscillating turret with cam rotation speed $120 \mathrm{r} / \mathrm{min}$

\subsection{Dynamic simulation}

The cam is made a rotary motion, the rotational speeds are $600 \mathrm{r} / \mathrm{min}$ and $120 \mathrm{r} / \mathrm{min}$ respectively, and a load with $500 \mathrm{~N} \cdot \mathrm{m}$ is performed on the oscillating turret. And then the cam mechanism starts to carry on the dynamics simulation, and the simulation end time is $0.1 \mathrm{~s}$, the steps are 1000 . During the process of the cam simulation, we can observe the real-time visualization, which can display high quality realistic picture was executed under the simulation and the result can be received as the same as real motioning. When the simulation is finished, the angular displacement, angular velocity and angular acceleration of oscillating turret with two kinds of cam rotational speeds are measured by using the post processing module of ADAMS. The results are shown in 
Fig. 6 and Fig. 7. Fig. 6 shows the simulation results with cam rotation speed $600 \mathrm{r} / \mathrm{min}$, and Fig. 7 shows the simulation results with cam rotation speed $120 \mathrm{r} / \mathrm{min}$. From simulation results, the actual angular displacement curve of oscillating turret is smooth, and it is consistent with the theoretical output curve of modified sine cam cure, which illustrates that the dynamic model of globoidal cam mechanism is correctly built. The actual angular velocity curve of the oscillating turret has a slight oscillation and the actual angular acceleration curve has a big fluctuation. There are many reasons for the results. On the one hand, it is due to impact of mechanism stiffness and external load. On the other hand, the error caused by the transformation from UG model to ADAMS model. At the same time, if the cam speed is reduced the actual maximum acceleration will be fallen down by comparing the actual output curve of the two kinds of speed. Therefore, the input speed of globoidal cam mechanism is an important factor that affects the output performance of the mechanism.

\section{Conclusion}

The globoidal cam mechanism with oscillating and dual stopping followers is widely used in many types of automatic production line, but the introduction of comprehensive analysis is few for working surface. In this paper, the equation of the working surface is derived by using the method of offset surface, which greatly simplifies the process of solving and analyzing. The virtual prototype system framework of the cam mechanism is established. The three-dimension digital model of the cam is built with UG. Dynamic simulation analysis of the cam mechanism is studied by means of software ADAMS. By dynamic simulation, the data of cam follower's displacement, velocity and acceleration are obtained. It is can be conclude that cam rotation speed makes great effects on the performance to the cam mechanisms.

\section{Acknowledgement}

This research was financially supported by the Key Research Projects of Shandong Province in China (No. 2015GGX103005 and No. 2016GNC110025) and the National Natural Science Foundation of China (No. 5130 5164). In addition, valuable suggestions from the reviewers in improving the quality of the paper are highly appreciated.

\section{References}

1. R. L. Norton. Cam design and manufacturing handbook,2nd Ed ( Industrial Press, New York, 2009)

2. V. Thinh Nguyen, D Joong Kim. Flexible cam profile synthesis method using smoothing spline curves, Mech Mach Theory, 42, 825-838 (2007)

3. S Acharyya, T Kanti Naskar. Fractional polynomial mod traps for optimization of jerk and hertzian contact stress in cam surface, Computers \& Structures, 86, 322-329 (2008)

4. H Qiu, C Jun Lin, Z Ye Li, et al. A universal optimal approach to cam curve design and its applications, Mech Mach Theory, 40, 669-692 (2005)

5. H S Yan, M C Tsai, M H Hsu. A variable speed method for improving motion characteristics of camfollower systems, ASME J Mech Des, 118, 250-257 (1996)

6. Y A Yao, H S Yan, C Zhang. Integration design of cam mechanism and servo control system, China Sci, 30, 524-530 (2000)

7. H.M. Lankarani and P.E. Nikravesh. Continuous Contact Force Models for Impact Analysis in Multibody Systems, Nonlinear Dynamics, 5, 193207 (1994)

8. James B. McConville. Introduction to Mechanical System Simulation Using Adams (SDC Publications, 2015) 